\title{
Age-structured vectorial capacity reveals timing, not magnitude of within-mosquito dynamics is critical for arbovirus fitness assessment
}

\author{
E. Handly Mayton ${ }^{1}$, A. Ryan Tramonte ${ }^{1}$, Helen J. Wearing ${ }^{2}$ and Rebecca C. Christofferson ${ }^{1,3^{*}}$ (i)
}

\begin{abstract}
Background: Transmission dynamics of arboviruses like Zika virus are often evaluated by vector competence (the proportion of infectious vectors given exposure) and the extrinsic incubation period (EIP, the time it takes for a vector to become infectious), but vector age is another critical driver of transmission dynamics. Vectorial capacity (VC) is a measure of transmission potential of a vector-pathogen system, but how these three components, EIP, vector competence and vector age, affect VC in concert still needs study.

Methods: The interaction of vector competence, EIP, and mosquito age at the time of infection acquisition $\left(\right.$ Age $_{\text {acquisition }}$ ) was experimentally measured in an Aedes aegypti-ZIKV model system, as well as the age-dependence of probability of survival and the willingness to bite. An age-structured vectorial capacity framework $\left(\mathrm{VC}_{\text {age }}\right)$ was then developed using both $\mathrm{EIP}_{\mathrm{Min}}$ and $\mathrm{EIP}_{\text {Max' }}$ defined as the time to first observed minimum proportion of transmitting mosquitoes and the time to observed maximum proportion of transmitting mosquitoes.

Results: The within-mosquito dynamics of vector competence/EIP were not significant among treatments where mosquitoes were exposed at different ages. However, $V C_{\text {age }}$ revealed: (i) age-dependence in vector-virus interactions is important for transmission success; (ii) lower vector competence but at shorter EIPs was sufficient for transmission perpetuation; and (iii) $R_{0}$ may be overestimated by using non-age-structured VC.

Conclusions: The results indicate that ultimately the temporal component of the virus-vector dynamics is most critical, especially when exposure occurred at advanced mosquito age. While our study is limited to a single virus-vector system, and a multitude of other factors affect both vector competence and mosquito mortality, our methods can be extrapolated to these other scenarios. Results indicate that how 'highly' or 'negligibly' competent vectors are categorized may need adjustment.
\end{abstract}

Keywords: Extrinsic incubation period, EIP, Vector competence, Aedes aegypti, Vectorial capacity, Zika, Arbovirus, Mortality, Biting rate

(C) The Author(s) 2020. This article is licensed under a Creative Commons Attribution 4.0 International License, which permits use, sharing, adaptation, distribution and reproduction in any medium or format, as long as you give appropriate credit to the original author(s) and the source, provide a link to the Creative Commons licence, and indicate if changes were made. The images or other third party material in this article are included in the article's Creative Commons licence, unless indicated otherwise in a credit line to the material. If material is not included in the article's Creative Commons licence and your intended use is not permitted by statutory regulation or exceeds the permitted use, you will need to obtain permission directly from the copyright holder. To view a copy of this licence, visit http://creativeco mmons.org/licenses/by/4.0/. The Creative Commons Public Domain Dedication waiver (http://creativecommons.org/publicdomain/ zero/1.0/) applies to the data made available in this article, unless otherwise stated in a credit line to the data. 


\section{Background}

The transmission dynamics of arboviruses such as Zika virus (ZIKV) are evaluated over several characteristics, namely vector competence and the extrinsic incubation period (EIP). Aedes aegypti is the primary vector of ZIKV and several studies have evaluated its competence to transmit the virus [1-4]. Vector competence is the ability of a mosquito to acquire and ultimately transmit a virus $[5,6]$. The time it takes for this process to occur is referred to as the extrinsic incubation period (EIP) [7]. Vector competence and EIP are interrelated measures of the proportion of vectors that become infectious given exposure and the time it takes for a vector to become infectious given exposure, respectively $[6,8]$. Thus, EIP can be described as the temporality of vector competence and has been used to evaluate the relative fitness of arbovirus systems [6, 8, 9]. In addition, EIP and vector competence are influenced by many parameters including vector species, mosquito-immune system, microbiota fauna, discrete populations within species, and environmental factors [2, 3, 5, 10-15].

Indeed, changes in arbovirus fitness and thus transmission dynamics have been predicated on altered vector competence, especially as a critical component of vectorial capacity [6, 16-24]. The composite of vector competence and EIP into a single, dynamic measure allows for a more comprehensive understanding of this process [5, 10, 25-27]. Not all mosquitoes that are exposed will be able to transmit (vector competence) and the time it takes for those mosquitoes that will transmit is not a constant (EIP), and so understanding this composite over several days post-infection is critical $[6,28]$.

Vectorial capacity (VC) was derived as a measure of transmission potential of a vector-borne pathogen by a competent vector, and incorporates both vector competence and EIP $[6,7,17,18,25,29]$. VC is the vectorcentric component of the basic reproduction number $\left(\mathrm{R}_{0}\right)$, and $\mathrm{VC}$ represents the number of secondary cases resulting from the introduction of a single infectious human individual per infectious day of that human index case $[30,31] . \mathrm{VC}$ is given by:

$$
V C=\frac{m a^{2} b p^{N}}{-\ln (p)}
$$

where ' $m$ ' is the density of mosquitoes relative to humans, ' $\mathrm{a}$ ' is the biting rate, ' $\mathrm{b}$ ' is the vector competence of the mosquito for a particular virus, ' $\mathrm{N}$ ' is the extrinsic incubation period, and ' $\mathrm{p}$ ' is the daily probability of mosquito survival [6]. And $\mathrm{R}_{0}$ is given by:

$$
R_{0}=\frac{c}{r} * V C
$$

where ' $r$ ' is the human recovery rate, or the reciprocal of the average human infectious period, and ' $c$ ' is the probability of transmission from the human to the vector given contact. $R_{0}$ is a threshold parameter, whereby an $R_{0}$ value $<1$ is not expected to sustain transmission and an $R_{0}$ value $\geq 1$ is likely to result in an outbreak $[11,32]$.

$\mathrm{VC}$ uses an average daily probability of survival to calculate the probability of a mosquito living through the $\operatorname{EIP}\left(\mathrm{p}^{\mathrm{N}}\right)$ and $\mathrm{p}^{\mathrm{N}} /(-\ln (\mathrm{p})$ represents the expected infectious days given $\mathrm{N}$ and $\mathrm{p}$ [33]. It is intuitive how mortality could impact transmission, but most studies address mortality based on a constant age at which the mosquito acquires the infection $[9,10,12,34,35]$. Mathematical modeling studies have addressed age structure with respect to EIP or bite-structure, but no studies have empirically investigated all three processes simultaneously $[9,36]$. Thus, herein, we experimentally test the hypothesis that transmission dynamics of arboviruses are as or more affected by time as a function of age versus time as a function of days post-infection. We further develop an age-structured vectorial capacity equation $\left(\mathrm{VC}_{\mathrm{age}}\right)$ to quantify these potential effects.

\section{Methods}

We first wanted to determine if age and/or prior blood meals affected the within-mosquito viral dynamics, as well as various life traits of the mosquito. To that end, we designated three treatment groups: YOUNG, OLDER and S.OLDER. The YOUNG group was offered an infectious blood meal at approximately 5 days postemergence (dpe). The OLDER group was offered a noninfectious blood meal at 5 dpe and then an infectious blood meal 1 week later (12 dpe). The S.OLDER group was not offered a prior, non-infectious blood meal, but a single infectious blood meal at approximately 12 dpe (to match the OLDER timing). S refers to sugar, which was done in order to distinguish the absence of a blood meal at 5 dpe. All non-infectious blood meals are referred to as "mock" blood meals, as they contain non-infectious cell culture media in place of infectious media.

\section{Virus and mosquitoes}

ZIKV strain PRVABC59 (Asian lineage), originally isolated from a human patient in Puerto Rico in 2015, was provided by Dr Barbara Johnson at the USA Centers for Disease Control and Prevention (GenBank: KU501215). The viral stock was passaged three times in Vero cells before passage on Vero cells for mosquito exposure. 
Supernatant was collected 3 days post-inoculation and titer determined as previously described [37]. The titer of ZIKV was verified by qRT-PCR at approximately $8 \times 10^{7}$ plaque forming units $(\mathrm{pfu}) / \mathrm{ml}$, matched across all exposure experiments. Virus used for mosquito exposure was never frozen, as this has been shown to negatively affect vector competence when compared to freshly cultured virus [38, 39]. Colony Aedes aegypti (Rockefeller) were provided by Dr Daniel Swale of the LSU Entomology Department, Baton Rouge, Louisiana, USA. To isolate the effect(s) of age, mosquitoes were maintained at constant conditions with 16:8 h light:dark periods, approximately $80 \%$ humidity (monitored using a digital $\mathrm{RH}$ monitor), and at $28{ }^{\circ} \mathrm{C}$ constant temperature. Mosquitoes were supplied with a $10 \%$ sucrose water solution after emergence via soaked cotton pledgets, which were replenished every $24 \mathrm{~h}$. Sucrose solution was removed $24 \mathrm{~h}$ before experiments and was again provided after blood feeding/mock starvation.

\section{Blood-feeding and oral exposure of Ae. aegypti}

Infectious and non-infectious blood meals were prepared with ZIKV-infected cell culture supernatant and non-infected cell culture supernatant, respectively. Whole bovine blood in Alsever's solution (Hemostat Labs, Dixon, CA, USA) was used in a 2:1 blood to supernatant ratio [28]. Mosquitoes were fed via the Hemotek membrane feeding system (Hemotek, Blackburn, UK) for $45 \mathrm{~min}$, after which mosquitoes were cold anesthetized and blood-fed females were placed into clean cartons until further treatment. Because starvation and cold anesthetization could affect mortality rates, every treatment was subjected to the same schedule of each of these conditions. For example, all groups were starved $24 \mathrm{~h}$ prior to days 5 and 12 post-emergence, regardless of whether that group would receive a blood meal. In addition, not only were blood-fed mosquitoes cold anesthetized and moved to a new carton, but other groups that did not feed on that schedule were treated the same in order to mimic these conditions and control for these factors. The experimental design is depicted in Additional file 1: Figure S1.

\section{Within-mosquito kinetics}

In order to determine if the timing of the infectious blood meal affected the within-mosquito viral kinetics, mosquitoes were sampled at 5, 8 and 11 days postinfection (dpi) to test for infection and dissemination across all groups (sample sizes are provided in Additional file 1: Table S1). Mosquito legs and bodies were placed into separate tubes containing $900 \mu \mathrm{l} \mathrm{BA-1} \mathrm{dilu-}$ ent media and BBs. Samples were then homogenized twice at $25 \mathrm{~Hz}$ for 3 min using a Qiagen Tissuelyzer (Qiagen, Hilden, Germany). RNA was extracted $(5 \times$ MagMax 96 Viral RNA Isolation Kit; Applied Biosystems, Foster City, CA, USA) and tested for the presence of viral RNA via qRT-PCR (SuperScript III One-Step RT-PCR System; Invitrogen, Carlsbad, CA, USA) as in [40]. Each treatment was repeated a total of three times and data was averaged over these replicates. Differences among the treatment groups for infection and dissemination rates were tested by a chi-square test for multiple proportions on 5,8 and $11 \mathrm{dpi}$.

\section{Transmission assay}

To directly assess transmission potential, 20 mosquitoes per treatment were force-salivated. Briefly, ZIKV-exposed mosquitoes were immobilized on ice before removing legs and wings. Mosquitoes were then placed on double-sided tape, and the proboscis of each mosquito was placed into a pipette tip containing $35 \mu \mathrm{l} \mathrm{FBS} \mathrm{with} 3 \mathrm{mmol} / \mathrm{l}$ ATP for $30 \mathrm{~min}$, as previously described [41]. Contents of the pipette tip were ejected into $100 \mu \mathrm{l} \mathrm{BA}-1$ diluent and stored at $-80{ }^{\circ} \mathrm{C}$ before testing (see below).

Mosquitoes from the two OLDER groups were sampled at 5,8 and $11 \mathrm{dpi}$, as well as $16 \mathrm{dpi}$ to represent the end of the mortality study ( $28 \mathrm{dpe})$. In order to characterize the EIP across the mosquito's lifetime, mosquitoes in group YOUNG were sampled at 5, 8 and $11 \mathrm{dpi}$, as well as additional days post-infection in order to match the age at which the older groups were sampled. Thus, by adding 7 to the time points in the older groups, we additionally sampled the YOUNG group at 12, 15 and $18 \mathrm{dpi}$, as well as 23 dpi which corresponds to the oldest time point of 28 days old. Samples were tested for the presence of viral RNA in saliva using the techniques described above $[40,41]$. Differences among the treatment groups' transmission rates were tested by Chi-square test for multiple proportions at each sampling timepoint.

\section{Mortality study}

Mortality studies were performed for the same three treatments (YOUNG, OLDER, and S.OLDER). We added additional mock blood meal controls (that is, accounting for any infectious blood meal-associated alteration of mortality) where a mock blood meal was used in place of infectious blood meals. The three controls were: (i) a mock blood meal at 5 dpe (M.Y) to correspond to the YOUNG treatment; (ii) a mock blood meal at 5 dpe, followed by another mock blood meal at 12 dpe (M.M) to correspond to the OLDER two blood meal treatment; and (iii) a mock blood meal at 12 dpe (S.M) to correspond to the S.OLDER, one blood meal treatment. An additional negative control treatment 
was performed where the mosquitoes were never blood-fed (S). All treatments were cold anesthetized at 5 and 12 dpe, regardless of whether they were offered a blood meal so that all mosquitoes experienced the same treatment, and mosquito density per carton was kept relatively constant with an average of 47 mosquitoes/ carton (range 36-58). Each mortality treatment was repeated a total of three times and data are averages of the three replicates.

Cartons were checked daily and, when present, dead mosquitoes were counted and removed up to 28 dpe (approximately 1 month), as this has been shown to be the upper limit of field survival of Ae. aegypti and is similar to the range used in Tesla et al. [13, 41]. Only mosquitoes that took all offered blood meals were included in the mortality analyses.

To test for differences in mortality rates among treatments, Kaplan-Meier survival analyses were conducted and the average time to death (TTD) was estimated. Daily mortality estimates relative to age were then predicted using best fits in $\mathrm{R}$ (version 3.5.2) [42].

\section{Determining age-structured willingness to probe}

One of the most influential parameters for determining transmission dynamics of vector-borne pathogens is the biting rate (because it impacts vector-host transmission in both directions). Biting rate is sometimes parameterized as the reciprocal of the number of days between feeds [43]. This assumes that the waiting time between bites is exponentially distributed, and we make this assumption for three biting rates: 0.5 (once every two days); 1 (once a day); and 2 (twice a day) [44]. However, we wanted to determine if the willingness of the mosquito to probe was affected by (i) timing of infectious blood meal and/or (ii) age of the mosquito [36]. While mosquito biting is a function of many factors, it has been shown that heat cues are sufficient to initiate host-seeking behaviors [45]. We use this to determine the willingness or probability of a mosquito probing, which can lead to transmission [46-48].

Ten to twelve mosquitoes per each ZIKV-infected treatment (YOUNG, OLDER and S.OLDER) were placed in individual, clear plastic canisters (Bioquip, Rancho Dominquez, CA, USA) $24 \mathrm{~h}$ before being provided a blood meal via a membrane feeder using $1 \mathrm{ml}$ discs with $800 \mu \mathrm{l}$ of blood (Hemotek, Blackburn, UK). This was done at the same dpi schedule as the vector competence studies above. Willingness to bite was assessed using a two-tiered approach by a single observer to control for observation bias. First, mosquitoes were observed through the clear canister for their general position in the canister and secondly, the disc was removed to determine if they were on or near the mesh at the top of the canister.
In all cases, these two methods of observation matched. That is, if a mosquito was observed to be at the blood meal prior to disc removal (looking through the canister), it did not move to the bottom of the canister upon disc removal.

This observation was performed at 1, 20 and $45 \mathrm{~min}$ post-placement of the disc and the disc was replaced between observation time points. Thus, a mosquito was assessed as "landed" and recorded as " 1 " if the female was at the top of the canister at any of the observation times. The female was otherwise classified as "not landed" and coded as " 0 " if she was at the bottom of the canister for all three observation times. We then calculated the probability of biting ( $\mathrm{Z}$ ) as a function of age. Z(age) was determined by fitting the proportion of mosquitoes that landed or fed at least once a day using a self-starting nonlinear least squares regression:

$$
Z(\text { age })=s+(g-s) \exp (-\exp (h) *(-a g e))
$$

where ' $\mathrm{s}$ ' is the asymptote, ' $\mathrm{g}$ ' is the zero-response parameter, and ' $h$ ' is the logarithmic rate constant. Comparison of probing differences among treatments was assessed both per day post-infection as well as per mosquito age by Kruskal-Wallis test.

\section{Age-structured vectorial capacity and $\mathrm{R}_{\mathbf{0}}$}

We re-formulated the vectorial capacity equation to estimate $\mathrm{VC}$ as a function of the age at which the mosquito acquires an infectious blood meal, redefining the parameters with respect to age at the time of acquisition of infection. We define Age acquisition as the age at which a mosquito acquires an infection (the day of taking the

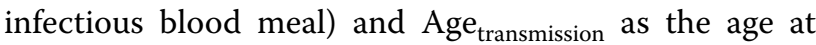
which the mosquito subsequently transmits (Fig. 1):

$$
\text { Age }_{\text {transmission }}=\text { Age }_{\text {acquisition }}+\text { EIP }
$$

In $\mathrm{VC}_{\text {age }}$ ' $\mathrm{m}$ ' is still the mosquito-to-human density and, for illustrative purposes, held constant here at 1 . The parameters $\mathrm{z}_{\text {acquisition }}$ and $\mathrm{z}_{\text {transmission }}$ are the probability of a mosquito biting at age of acquisition and age at time of transmission, respectively. The traditional calculation of $\mathrm{p}^{\mathrm{N}}$ represents the probability of a mosquito living through a fixed number of days $\mathrm{N}$, the EIP, and $\mathrm{p}^{\mathrm{N}}$ / $(-\ln (\mathrm{p}))$ represents the expected infectious days given $\mathrm{N}$ and $\mathrm{p}$ [33]. In the context of an age-dependent vectorial capacity framework, we can calculate a more precise probability of surviving based on the day the mosquito obtained an infection and the cumulative survival prob-

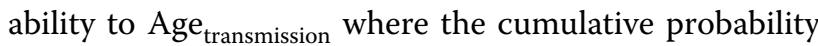
of living through the EIP given Age ${ }_{\text {acquisition }}$ is given by: 


$$
p\left(\text { surviving to Age } e_{\text {transmission }}\right)=\prod_{j=1}^{\text {Age }} \prod_{\text {transmission }} p_{j}
$$

where ' $\mathrm{p}_{\mathrm{j}}$ ' is the probability of daily survival on day $j$ post emergence. We further estimate the infectious period (L) in an age-structured way by numerically deriving L:

$$
L=T T D-\text { Age }_{\text {transmission }}
$$

where TTD is the average time-to-death derived from the experimental mortality study.

Using the data from our experimental studies, we calculated Age transmission $_{\text {for two scenarios: } \text { EIP }_{\text {min }} \text { and EIP }}$ max from our observed transmission data [49], and calculated $\mathrm{VC}_{\text {age }}$ as:

$$
V C_{\text {age }}=m b\left(z_{\text {acquisition }} a\right)\left(z_{\text {transmission }} a\right)\left(\prod_{j=1}^{\text {age }_{\text {transmission }}} p_{j}\right) L
$$

$\mathrm{VC}$ is a component of $\mathrm{R}_{0}$, which also includes the average infectious period of the human and the probability of transmission from the human to the vector. For illustrative purposes, we calculated $\mathrm{R}_{0}$ using both the traditional calculation of $\mathrm{VC}$ (Eq. 1), as well as $\mathrm{VC}_{\mathrm{age}}$. We made the following assumptions: mosquito density is held constant at $\mathrm{m}=1$; the average infectious period of the human is 5 days $\left(\mathrm{r}^{-1}\right)$ [50]; and the average probability of transmission from human to vector is parameterized experimentally as the average proportion of mosquitoes that develop a midgut infection given exposure (c). From these data, we can calculate the value of VC needed to push $\mathrm{R}_{0}$ above this threshold. We use this value as a "threshold" to compare $\mathrm{VC}_{\text {age }}$ across treatment groups and with the traditional VC measures.

All statistics and subsequent graphics were performed and generated using $\mathrm{R}$ version 3.5.2 [42]. All packages used are provided in Additional file 1: Text S1. All functions and function parameters used to fit the data and obtain age-dependent distributions of these parameters are given in Additional file 1: Table S2. Goodness-of-fit was assessed either through AIC (for non-linear models) or $R^{2}$ for linear models.

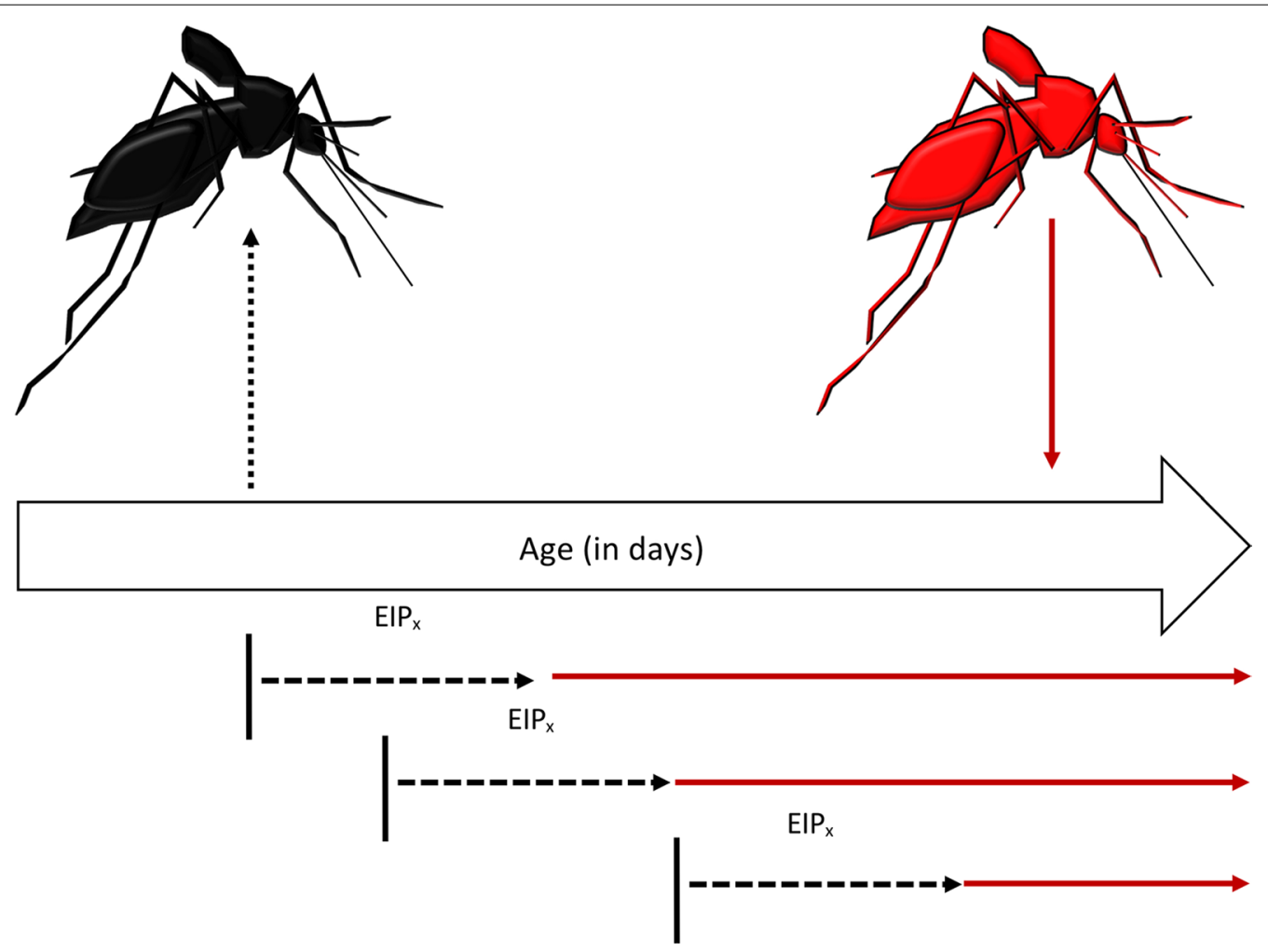

Fig. 1 Vector age at time of infection acquisition determines transmission opportunity. Uninfected mosquitoes (black, left) acquire an infection at Age $_{\text {acquisition }}$ (black vertical lines) and after a certain EIP (black dashed lines), a proportion will become infectious (red, right), but for a certain period of time (red horizontal lines) that is dependent on Age acquisition 


\section{Results}

Within-mosquito dynamics are more affected by time as a function of age versus days post-infection

We first wanted to determine if the age at which a mosquito is offered an infectious blood meal and acquires the ZIKV infection (Age acquisition ) impacted the withinvector kinetics of the mosquito. To do this, we looked at infection, dissemination, and transmission of the three treatments in the context of days post-infection. We found no significant difference in the infection $(P>0.05)$ or dissemination rates $(P>0.05)$ across all treatments (Additional file 1: Table S1). However, when direct age comparisons are made, the effect of Age $_{\text {acquisition }}$ on infection becomes obvious (Fig. 2). The average rate of

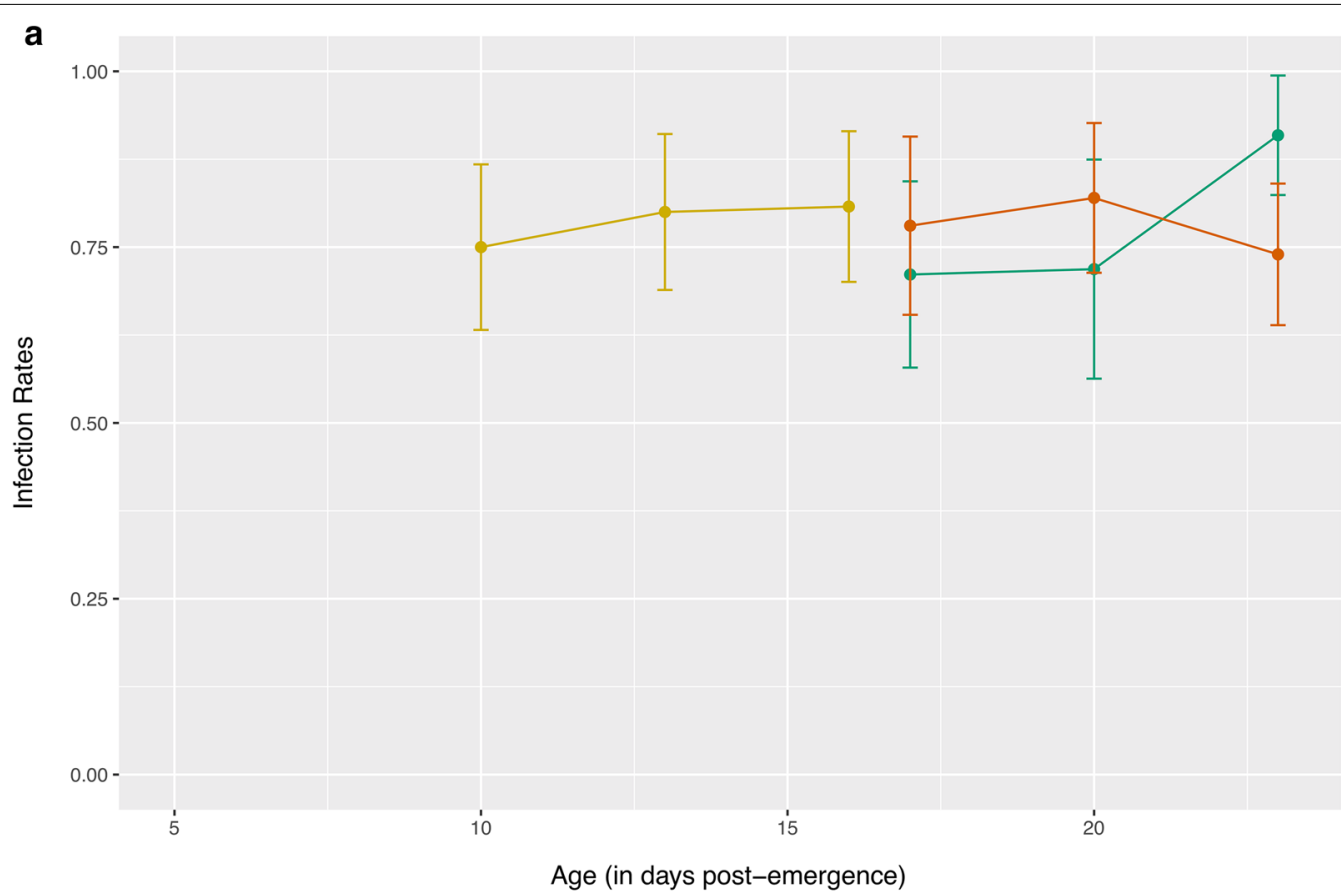

b 1.00

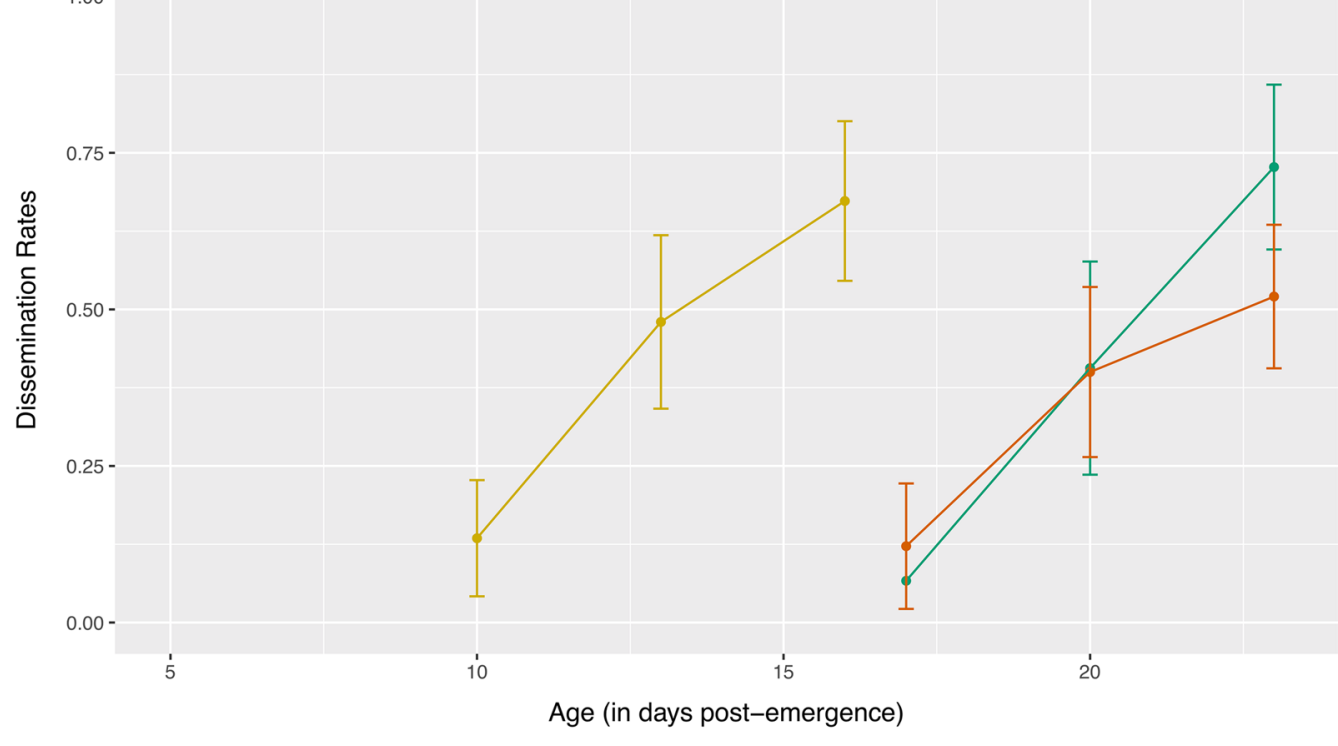

Treatment: $\rightarrow$ OLDER $\rightarrow$ S.OLDER $\rightarrow$ YOUNG

Fig. 2 Infection and dissemination rates in the context of mosquito age. Despite no significant effect of treatment on infection and dissemination rates when assessed over days post-infection, Age acquisition has an obvious impact on the timing of these processes 
infection of mosquitoes given exposure was $78.2 \%$ across all treatments and days post-infection (95\% confidence interval: 74.3-82.0\%).

When we evaluated the proportion transmitting in terms of days post-exposure (5, 8 and 11 only) none of the treatments were transmitting on days 5 or $8 \mathrm{dpi}$. On day 11 , the transmission rates were $5 \%, 10 \%$ and $15 \%$ in groups OLDER, S.OLDER and YOUNG, respectively. These differences were not statistically significant using the Chi-square test for proportions $(P>0.5)$ (Additional file 1: Table S1).

However, when we investigated mosquitoes from group YOUNG at time points that age-matched OLDER and S.OLDER treatments (Table 1), the YOUNG group achieved a maximum transmission of $45 \%$ at 28 days old (23 dpi) versus only $15 \%$ for the S.OLDER group and $10 \%$ for the OLDER group at 28 days old (16 dpi).

We define the EIP $_{\max }$ as the earliest EIP (days postinfection) where the maximum proportion of mosquitoes are transmitting, and we define the EIP ${ }_{\text {min }}$ as the earliest EIP where the proportion of mosquitoes transmitting is minimal, but greater than 0 . EIP values at different vector competence levels have been used to evaluate the vectorial capacity for malaria [49]. For the YOUNG group, EIP $_{\max }$ was $23 \mathrm{dpi}$ at $45 \%$, and $\mathrm{EIP}_{\min }$ was $11 \mathrm{dpi}$ at $15 \%$. For the OLDER group, EIP ${ }_{\max }$ was $16 \mathrm{dpi}$ at $10 \%$ and $\mathrm{EIP}_{\min }$ was $11 \mathrm{dpi}$ at $5 \%$. For the S.OLDER group, EIP ${ }_{\max }$ was $16 \mathrm{dpi}$ at $15 \%$ and the $\mathrm{EIP}_{\min }$ was $11 \mathrm{dpi}$ at $10 \%$.

Table 1 Transmission rates for each day post-infection (dpi) and corresponding mosquito age for each of the three treatment groups

\begin{tabular}{llll}
\hline Treatment & $\begin{array}{l}\text { dpi (day post- } \\
\text { infection) }\end{array}$ & $\begin{array}{l}\text { Age (day post- } \\
\text { emergence) }\end{array}$ & $\begin{array}{l}\% \text { Transmission } \\
(n=20)\end{array}$ \\
\hline YOUNG & 5 & 10 & 0 \\
& 8 & 13 & 0 \\
& 11 & 16 & 15 \\
& 12 & 17 & 15 \\
& 15 & 20 & 10 \\
& 18 & 23 & 35 \\
OLDER & 23 & 28 & 45 \\
& 5 & 17 & 0 \\
& 8 & 20 & 0 \\
& 11 & 23 & 5 \\
S.OLDER & 16 & 28 & 10 \\
& 5 & 17 & 0 \\
& 8 & 20 & 0 \\
& 11 & 23 & 10 \\
& 16 & 28 & 15 \\
\hline
\end{tabular}

Mortality of Aedes aegypti is modestly affected by timing of infectious blood meal

When each infectious treatment was compared to a matching non-infectious control group, the only significant difference in average time to death (TTD) was between the S.OLDER and S.M treatments, with an estimated difference in TTD of two days (Additional file 1: Table S3). Of interest, the non-blood-fed sugar-only controls died significantly faster than any of the blood-fed treatments with an average TTD of 19.6 days (Additional file 1: Table S3), which has been previously shown [51].

Pairwise comparisons of the ZIKV-exposed treatments determined that group YOUNG had a significantly longer average time to death (TTD) when compared to groups OLDER and S.OLDER, though this difference was modest (0.6 and 1.4 days, respectively) (Fig. 3). The TTD for the YOUNG group was 25.9 days, 25.3 days for the OLDER group, and 24.5 days for the S.OLDER group. This corresponds to average daily survival probabilities of $0.962,0.961$ and 0.960 for the YOUNG, OLDER and S.OLDER groups, respectively. Predicted daily survival rates were generated for the YOUNG group using a non-linear fit and a linear model was fit to the OLDER and S.OLDER groups. The parameters of these models and goodness-of-fit assessments are given in Additional file 1: Table S2, and the observed and predicted values are shown in Additional file 1: Figure S2 for all three treatment groups. Additional comparisons were made among the treatment and control groups, detailed in Additional file 1: Text S2, Figure S3 and Table S3.

\section{Age-dependence of willingness to probe}

There was no significant difference in the willingness to probe based on treatment (YOUNG, OLDER and S.OLDER) at each day post-infection via the KruskalWallis test, but there was a significant effect of age $(P<0.05)$. We then were able to fit a daily probability of probing based on age (Fig. 4).

\section{$\mathrm{VC}_{\text {age }}$ framework for assessing transmission as a function of age and effect on $\mathrm{R}_{\mathbf{0}}$}

To calculate $\mathrm{VC}_{\text {age }}$, we used treatment-group specific average TTD and predicted daily probabilities of survival (Additional file 1: Figure S4) and the overall daily prediction of willingness to bite in Fig. 4. Since biting rate (the number of bites per day) is a field-derived parameter, we calculated $\mathrm{VC}_{\text {age }}$ over three biting rates: 0.5 (once every 2 days), 1 (once a day), and 2 (twice a day) [28]. For comparison, we calculated the traditional VC using the average life-dependent traits determined experimentally above, $\mathrm{EIP}_{\min }$ and $\mathrm{EIP}_{\max }$, and the biting rates referenced 


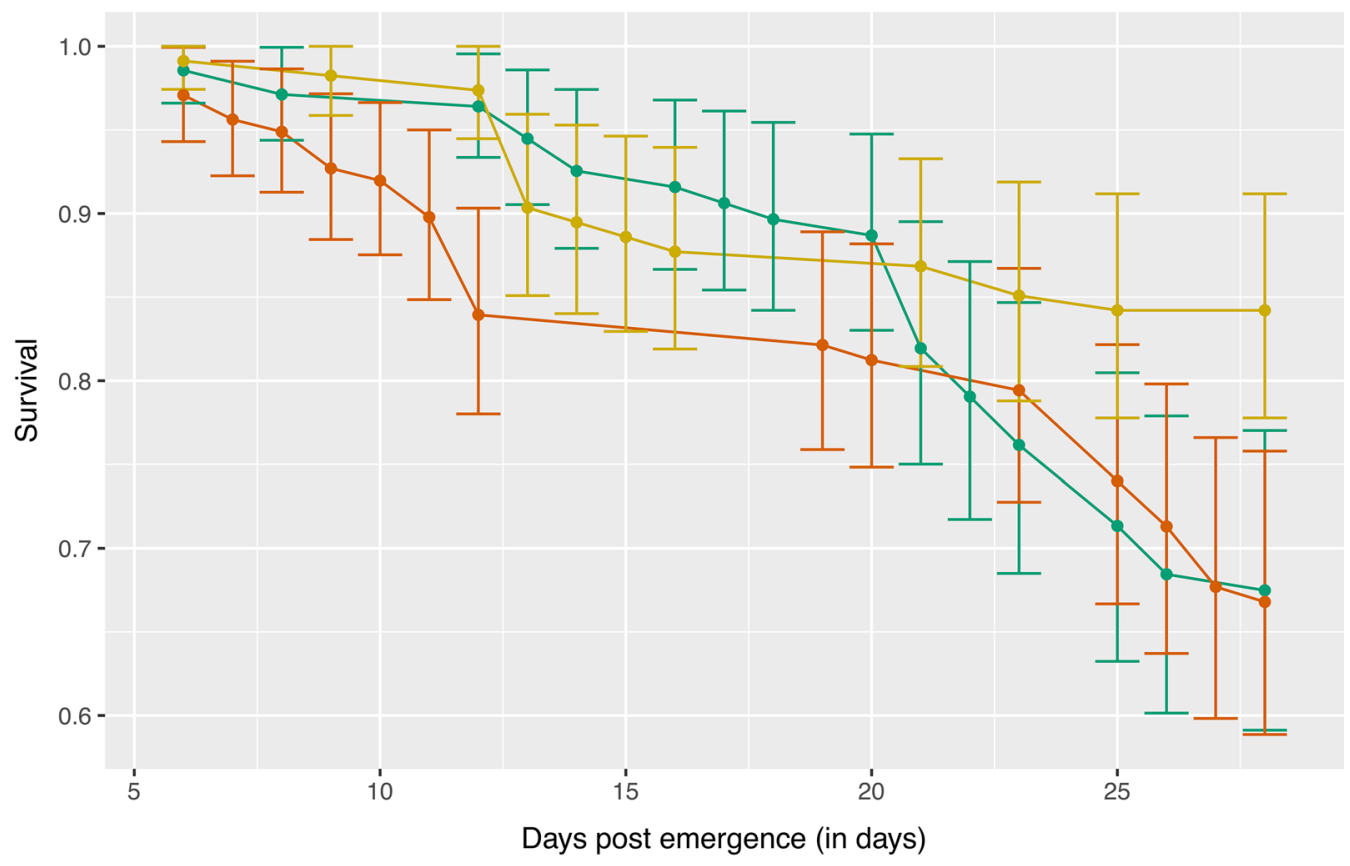

Treatment: $\rightarrow$ OLDER $\rightarrow$ S.OLDER $\rightarrow$ YOUNG

Fig. 3 Survival curves of female Ae. aegypti by treatment. Each line represents the combined data from three replicates per treatment: YOUNG (gold line), OLDER (green line), and sugar-OLDER (S.OLDER, red line). Average time to death of treatment, YOUNG was significantly, but modestly, longer than treatments OLDER and S.OLDER

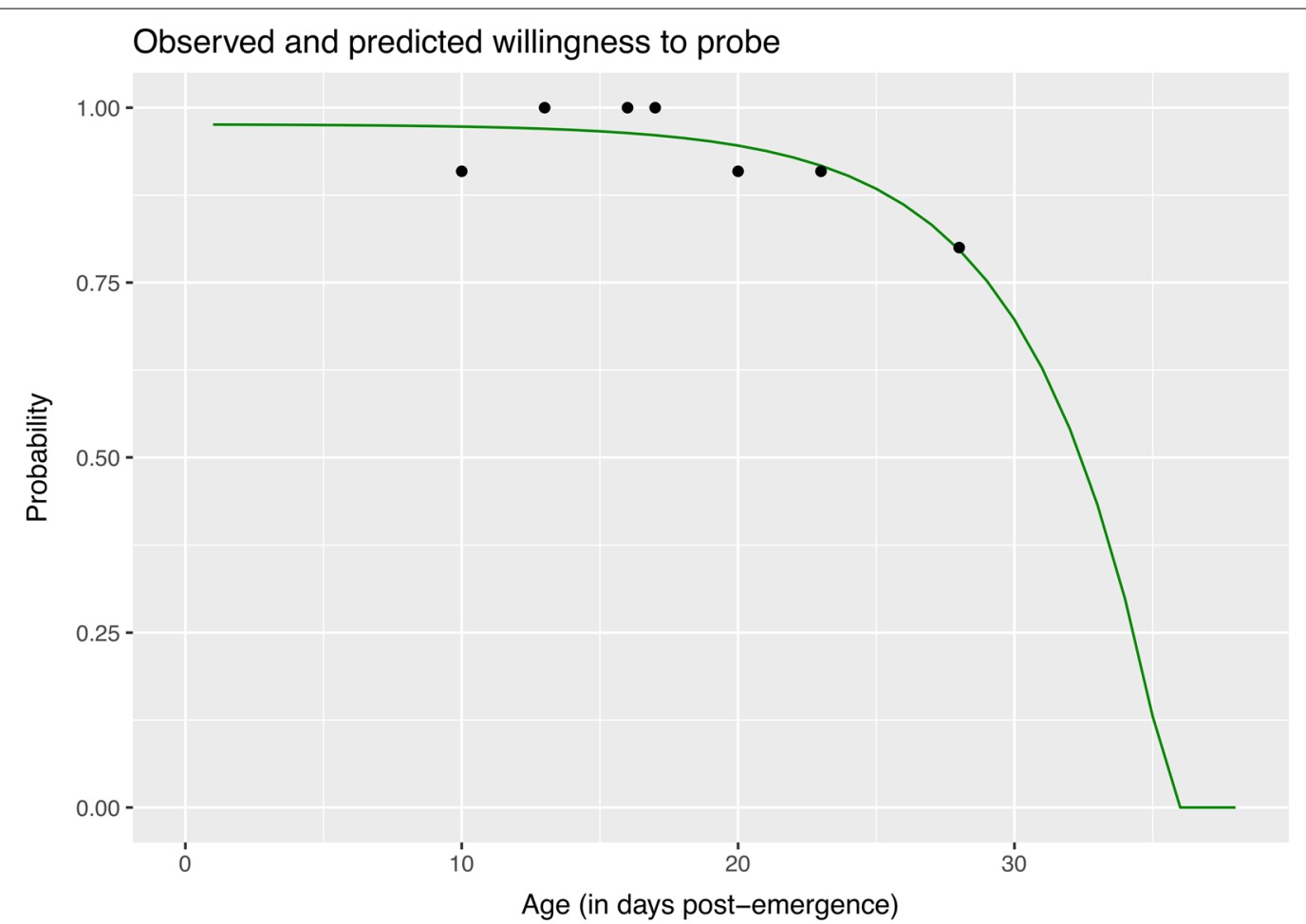

Fig. 4 Observed and predicted probabilities of daily biting. The observed daily biting frequencies (dots) from the laboratory experiments and the fitted daily predictions (green curve) 
Table 2 Traditional VC calculations for EIP $_{\min }$ and $\mathrm{EIP}_{\max }$ for each treatment group (calculated according to Eq. 1) and corresponding $\mathrm{R}_{0}$ (according to Eq. 2)

\begin{tabular}{|c|c|c|c|c|}
\hline Treatment & EIP & Biting rate & VC & $\mathrm{R}_{0}$ \\
\hline \multirow[t]{6}{*}{ YOUNG } & \multirow[t]{3}{*}{$\mathrm{EIP}_{\max }$} & 2 & 19.06 & 74.52 \\
\hline & & 1 & 4.77 & 18.65 \\
\hline & & 0.5 & 1.19 & 4.65 \\
\hline & \multirow[t]{3}{*}{$E P_{\min }$} & 2 & 10.11 & 39.53 \\
\hline & & 1 & 2.53 & 9.89 \\
\hline & & 0.5 & 0.632 & 2.47 \\
\hline \multirow[t]{6}{*}{ OLDER } & \multirow[t]{3}{*}{$\mathrm{EIP}_{\max }$} & 2 & 4.03 & 15.76 \\
\hline & & 1 & 1.01 & 3.95 \\
\hline & & 0.5 & 0.25 & 0.978 \\
\hline & \multirow[t]{3}{*}{$E I P_{\min }$} & 2 & 6.49 & 26.98 \\
\hline & & 1 & 1.62 & 6.33 \\
\hline & & 0.5 & 0.41 & 1.60 \\
\hline \multirow[t]{6}{*}{ S.OLDER } & \multirow[t]{3}{*}{$E I P_{\max }$} & 2 & 7.65 & 29.91 \\
\hline & & 1 & 1.91 & 7.47 \\
\hline & & 0.5 & 0.48 & 1.88 \\
\hline & \multirow[t]{3}{*}{$E I P_{\min }$} & 2 & 6.25 & 24.44 \\
\hline & & 1 & 1.56 & 6.10 \\
\hline & & 0.5 & 0.39 & 1.52 \\
\hline
\end{tabular}

above. These VC values are given in Table 2, along with corresponding values for $\mathrm{R}_{0}$. In the absence of published data regarding the probability of human to mosquito ZIKV transmission success, we used the average infection rate from our experimental data $(78.2 \%)$ to parameterize human to mosquito transmission, recognizing that this model system used a high dose of ZIKV titer, although such titers in individuals are not unheard of $[52,53]$.

\section{YOUNG group}

To achieve an $R_{0}$ of 1 , the vectorial capacity needed to be at or above 0.256 . When parameterized with EIP max $_{\text {, }}$ the $\mathrm{VC}_{\text {age }}$ at a biting rate of 0.5 (once every 2 days) did not achieve this minimal value, but at biting rates of once or twice per day, $\mathrm{VC}_{\text {age }}$ was sufficient to push $\mathrm{R}_{0}$ over the threshold of one (Fig. 5). We next determined the window of opportunity, which encompasses the days postemergence when a mosquito acquires an infection and which results in $V_{\text {age }}$ values where $R_{0}$ would be greater than one. The window of opportunity when we parameterized $\mathrm{VC}_{\mathrm{age}}$ with $\mathrm{EIP}_{\max }$ indicated that a virus must be acquired within the first 2 days post-emergence when the bite rate was twice or once per day (Fig. 5). Interestingly, when parameterized with EIP $_{\text {min }}$, all bite rates reached sufficient $\mathrm{VC}_{\mathrm{age}}$ values. The window of opportunity was
14 days post-emergence for a bite rate of twice a day, 12 days post-emergence for a bite rate of once a day, and 7 days post-emergence for a bite rate of once every 2 days (Fig. 5). Even at its highest, $\mathrm{VC}_{\mathrm{age}}$ indicates that traditional $\mathrm{VC}$ calculations are likely overestimates.

\section{OLDER and S.OLDER groups}

For both the OLDER and S.OLDER groups, the infectious blood meal occurred at 12 days post-emergence, which was outside the window of opportunity for $\mathrm{VC}_{\mathrm{age}}$ in all but one scenario (Additional file 1: Figure S4). Only at a bite rate of twice daily did the OLDER group achieve the minimum $\mathrm{VC}_{\text {age }}$ of 0.256 on or after Age acquisition of 12 days-old, and only when parameterized with the EIP ${ }_{\text {min }}$. The window of opportunity for this scenario was days 12-13 post-emergence.

With these vector age-dependent traits, both EIP and vector competence would need to be altered significantly to achieve a $\mathrm{VC}_{\text {age }} \geq 0.256$ at 12 days post-emergence for other scenarios. For example, we consider a hypothetical EIP $\mathrm{max}_{\text {max }}$ of $\mathrm{EIP}_{50}$ (the time it takes for $50 \%$ of exposed mosquitoes to transmit) and a hypothetical EIP ${ }_{\text {min }}$ of $\mathrm{EIP}_{10}$ to demonstrate how these group-specific mortality rates and the age structure of willingness to bite interact to drive vectorial capacity above the threshold in the context of Age $_{\text {acquisition }}[13,49,54]$. The S.OLDER group would need a maximum $\mathrm{EIP}_{50}$ of $12 \mathrm{dpi}$ or $11 \mathrm{dpi}$ for biting rates of twice and once daily, respectively, and $8 \mathrm{dpi}$ for a biting rate of once every 2 days. For the OLDER group, the maximum $\mathrm{EIP}_{50}$ would need to be 13,12 , or $11 \mathrm{dpi}$ for biting rates of twice or once daily, or once every 2 days, respectively. With the EIP $_{\text {min }}$, similar maximum EIPs were noted of 12 and 9 dpi for biting rates of twice or once daily, respectively. For both the OLDER and S.OLDER groups, with a hypothetical EIP ${ }_{\min }$ of $10 \%$, $\mathrm{VC}_{\text {age }}$ never got above the threshold needed to get $\mathrm{R}_{0}$ at or above 1.

\section{Discussion}

Prediction of vector-borne disease spread remains difficult, as transmission of vector-borne disease is a dynamic, multifaceted system. This includes life traits of the mosquito, environmental factors, and vector-virus interactions $[14,16,23,55,56]$. Here we demonstrate through a combination of experimental and computational methods that mosquito age at the time of pathogen acquisition is a powerful driver of transmission potential due, in large part, to the age-dependence of daily mortality and biting habits. Further, these drivers lead to differences in the estimates of $R_{0}$.

Our study, which focused upon the age at the time of infectious blood meal, showed no significant impacts on the vector competence of colony Ae. aegypti for ZIKV. 


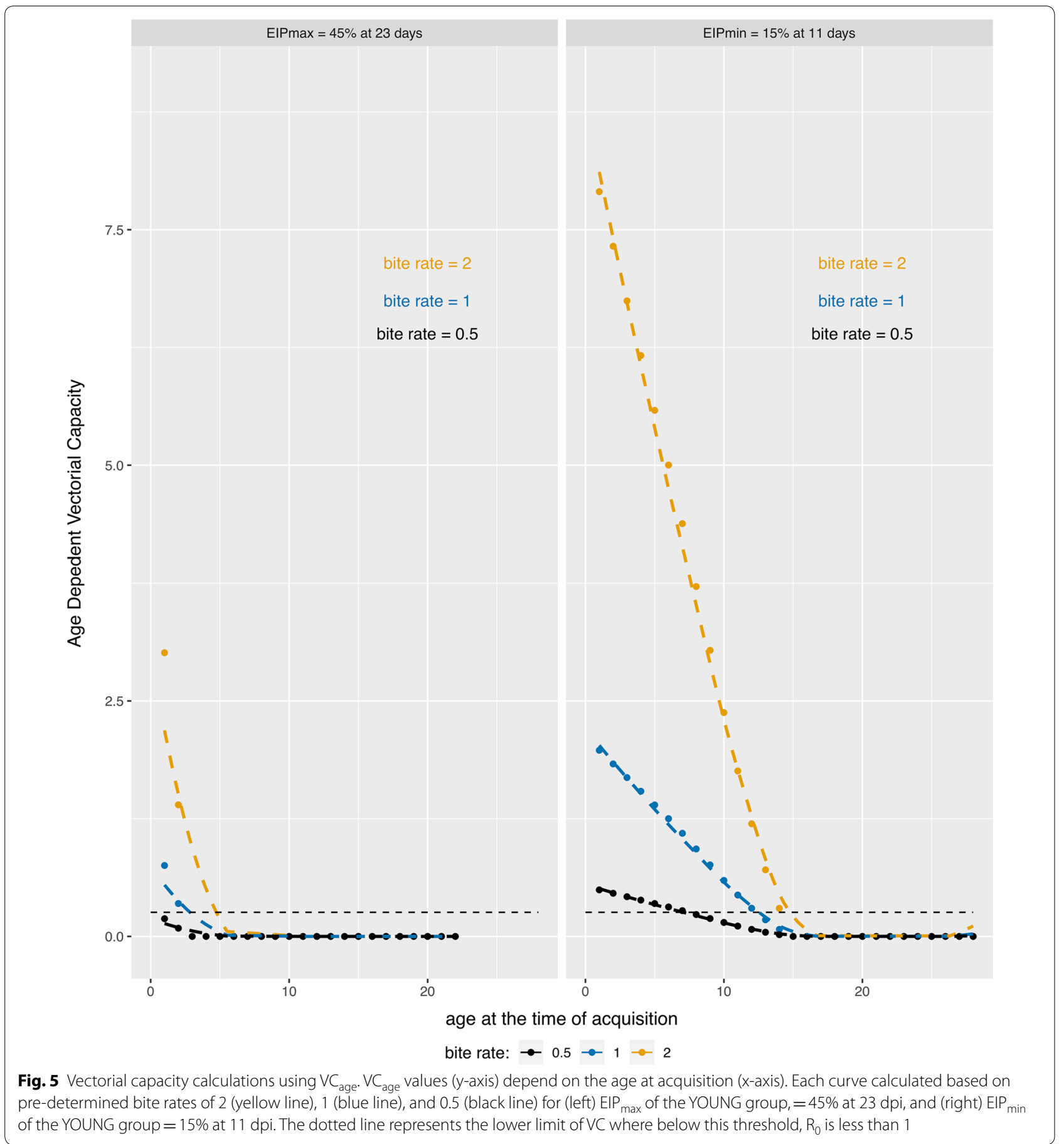

While a recent study did show there were significant effects of multiple blood meals on vector competence [57], we delivered the second blood meal at a much longer interval (7 days versus 3 dpi in [57]), which likely plays a role in this disparate finding and indicates that there may be age-dependence in this phenomenon as well. However, this hypothesis is outside the scope of the present study. In our mortality study, we did observe a modest difference in TTD. We also observed a very short TTD in mosquitoes only given sugar with no blood meal. We chose not to pursue these effects, as the differences in TTD were modest and the impacts of no blood meal have been previously observed. 
The technology for determining the age structure of natural mosquito populations in the field is currently still in development. For example, a study using near-infrared spectroscopy was able to predict the age of female Ae. aegypti \pm 2 days, indicating that determining the age structure of a mosquito population is possible, and that such technology could be refined for field studies [58, 59]. Further, mid-infrared spectroscopy had varying, but some promising results in determining the age structure of Anopheles mosquitoes [59, 60]. As these technologies are pursued and refined, there will be a need for ways to understand and quantify age-dependent interactions among vector competence, EIP, mosquito lifespan, and biting behavior $[16,61]$.

The methodologies in this study highlight the need to understand the quantitative interaction between vector competence, mosquito mortality, and age at time of infection acquisition. Thus, we anticipate that this methodology could be used to explore other important modifiers of vector competence and mosquito mortality and the interaction of the two, such as innate mosquito immunity response to infection, adult and larval microbiome, as well as other extrinsic factors known to affect these traits $[2,3,5,10-15]$. Several recent studies have focused on environmental factors such as temperature, and found that temperature not only affects vector competence of many arboviruses, especially in Aedes aegypti, but also several life traits of the mosquito $[41,55,56]$. This means that transmission is ultimately impacted by the interactions of all of these temporally dependent processes, and future studies should consider the age structure when assessing these impacts. Studies have also shown the impact of vector species, in particular the difference between field-derived and laboratory strains [2, 3]. Here, we used a laboratory strain of Ae. aegypti, which has been shown to vary in its competence when compared to a field-derived strain. Future use of $\mathrm{VC}_{\text {age }}$ could highlight these differences, as well as differences among various arbovirus-arthropod systems.

Our results demonstrate the importance of age structure when evaluating the fitness of a mosquito-virus system and indicate that $R_{0}$ may be overestimated when it is not considered. This framework further shows that how within-mosquito arbovirus fitness is measured, often by comparing proportions of transmitting mosquitoes at arbitrary time points, is not sufficient. Here, when the Age $_{\text {acquisition }}$ was advanced, the difference in hypothetical EIPs necessary for the system to succeed was not very different. For example, in the OLDER and S.OLDER groups, there was only one day difference between the $\mathrm{EIP}_{\text {min }}$ and $\mathrm{EIP}_{\max }$ needed for success at biting rates of 2 and 1, though these quantities represented a difference of $40 \%$ in the proportion of mosquitoes transmitting. The same trend was demonstrated in the YOUNG group where EIP ${ }_{\text {min }}$ resulted in longer windows of opportunity compared to EIP $_{\text {max }}$. Additionally, when the minimum time to transmission is shorter, a larger portion of the mosquito population contributes to transmission, because $\mathrm{VC}_{\text {age }}$ implicates older mosquitoes in this scenario given no significant reduction in mortality due to extrinsic factors [62]. Thus, $\mathrm{VC}_{\text {age }}$ reveals that the temporality of the withinmosquito arbovirus dynamics is more impactful than the ultimate magnitude of this widely used fitness measure. More investigations into the earlier and minimal transmission rates, such as at earlier time points that we did not consider, may be warranted to quantify fully the contribution of lower but faster dissemination profiles in arbovirus systems.

A study by Althouse et al. [63] also found that the temporality of transmission from non-human primates was sometimes more impactful than the magnitude of the viremia leading to transmission to the mosquito. They proposed a "tortoise-and-the-hare" (TatH) model to describe this relationship between arboviral viremia profiles in non-human primates and the predicted transmission success to vectors, showing that the strategy of "slow and steady" viremia, i.e. lower levels for longer periods, resulted in higher predicted transmission success of arboviruses [63]. This same TatH model recently described macro-transmission dynamics in Colombia, where it was demonstrated that slow burn-in epidemics actually resulted in cumulatively more cases and higher $R_{0}$ values than in initially explosive outbreaks [64]. Vector competence is a continuous process over time, and as such, EIP ${ }_{\text {min }}$ and $\mathrm{EIP}_{\max }$ are not independent measures. Thus, vector competence profiles with higher transmission rates at earlier times ("hare strategy") will always be more fit than lower proportions at the same EIP. However, this study demonstrates that in systems where the maximum measured vector competence is low, but the time to minimum transmission is short ("tortoise strategy") [65], there is still a good chance the system will succeed. $\mathrm{VC}_{\text {age }}$ also suggests that how arbovirus phenotypes are compared and ranked, and perhaps even how the field identifies 'highly' or 'negligibly' competent vectors, may need adjustment in the context of mosquito age.

\section{Conclusions}

We demonstrate that mosquito age may not affect experimentally determined infection, dissemination, or transmission rates when assessed in the traditional days post-infection manner. However, when we consider mosquito mortality, EIP, and vector competence in the context of a more holistic measure of transmission potential, 
vectorial capacity, and further adjust $\mathrm{VC}$ for the effect of age at the time of mosquito infection, we demonstrate that age can be an important factor, and that how some arbovirus fitness measures are assessed may need reconsideration.

\section{Supplementary information}

Supplementary information accompanies this paper at https://doi. org/10.1186/s13071-020-04181-4.

Additional file 1: Figure S1. Illustration of main treatment design for vector competence experimentation. Table S1. Infection and dissemination rates for each day post-infection (dpi) and corresponding mosquito age for each of the three ZIKV treatments. Table S2. Modeled fits of parameters, the type of model, and the parameter values. Figure S2. Observed and predicted daily probabilities of survival for the three treatment groups. Text S1. R packages used in the study. Text S2. Comparison of mortality of Aedes aegypti with respect to bloodmeals. Figure S3. Mortality curves for all treatments and associated controls. Table S3. Mean time to death and sample size for ZIKV-infection treatments and unexposed controls used in the mortality study. Figure S4. Demonstration of the $V_{\text {age }}$ for the two treatments exposed at older ages.

\section{Abbreviations}

EIP: extrinsic incubation period; ZIKV: Zika virus; VC: vectorial capacity; dpe: days post-emergence; dpi: days post-infection.

\section{Acknowledgements}

We would like to thank Dr. Barbara Johnson for providing our viral strain, as well as Dr. Daniel Swale for providing us with mosquito eggs. We would like to thank all our laboratory colleagues for putting up with the hogging of freezer space to hold all of the samples generated for this study. And last but not least, we'd like to thank Aesop for his apparently ubiquitously applicable fable.

\section{Authors' contributions}

EHM and RCC conceived and designed the study. EHM, ART and RCC performed the experiments. EHM, HJW and RCC carried out data analysis and interpretation. EHM, HJW and RCC wrote the manuscript. All authors read and approved the final manuscript.

\section{Funding}

This study was funded through NIH/NIGMS R01GM122077.

\section{Availability of data and materials}

All data generated or analyzed during this study are included in this published article and its additional file.

\section{Ethics approval and consent to participate}

Not applicable.

\section{Consent for publication}

Not applicable.

\section{Competing interests}

The authors declare that they have no competing interests.

\author{
Author details \\ ${ }^{1}$ Department of Pathobiological Sciences, School of Veterinary Medicine, \\ Louisiana State University, Baton Rouge, LA, USA. ${ }^{2}$ Departments of Biology \\ and Mathematics \& Statistics, University of New Mexico, Albuquerque, NM, \\ USA. ${ }^{3}$ Center for Computation and Technology, Louisiana State University, \\ Baton Rouge, LA, USA.
}

Received: 10 January 2020 Accepted: 10 June 2020 Published online: 15 June 2020

\section{References}

1. Costa-da-Silva AL, loshino RS, Araujo HR, Kojin BB, Zanotto PM, Oliveira DB, et al. Laboratory strains of Aedes aegypti are competent to Brazilian Zika virus. PLoS ONE. 2017;12:e0171951.

2. Roundy CM, Azar SR, Rossi SL, Huang JH, Leal G, Yun R, et al. Variation in Aedes aegypti mosquito competence for Zika virus transmission. Emerg Infect Dis. 2017;23:625-32.

3. Chouin-Carneiro T, Vega-Rua A, Vazeille M, Yebakima A, Girod R, Goindin D, et al. Differential susceptibilities of Aedes aegypti and Aedes albopictus from the Americas to Zika virus. PLoS Negl Trop Dis. 2016;10:e0004543.

4. Musso D, Gubler DJ. Zika virus. Clin Microbiol Rev. 2016;29:487-524.

5. Valderrama A, Diaz Y, Lopez-Verges S. Interaction of Flavivirus with their mosquito vectors and their impact on the human health in the Americas. Biochem Biophys Res Commun. 2017;492:541-7.

6. Kramer LD, Ciota AT. Dissecting vectorial capacity for mosquito-borne viruses. Curr Opin Virol. 2015;15:112-8.

7. Christofferson RC, Mores CN, Wearing HJ. Bridging the gap between experimental data and model parameterization for chikungunya virus transmission predictions. J Infect Dis. 2016;214(Suppl. 5):S466-70.

8. Ohm JR, Baldini F, Barreaux P, Lefevre T, Lynch PA, Suh E, et al. Rethinking the extrinsic incubation period of malaria parasites. Parasit Vectors. 2018;11:178.

9. Bellan SE. The importance of age dependent mortality and the extrinsic incubation period in models of mosquito-borne disease transmission and control. PLoS ONE. 2010;5:e10165.

10. Maciel-de-Freitas R, Koella JC, Lourenco-de-Oliveira R. Lower survival rate, longevity and fecundity of Aedes aegypti (Diptera: Culicidae) females orally challenged with dengue virus serotype 2 . Trans R Soc Trop Med Hyg. 2011;105:452-8.

11. Brady OJ, Godfray HC, Tatem AJ, Gething PW, Cohen JM, McKenzie $\mathrm{FE}$, et al. Vectorial capacity and vector control: reconsidering sensitivity to parameters for malaria elimination. Trans R Soc Trop Med Hyg. 2016;110:107-17.

12. Robert MA, Christofferson RC, Silva NJ, Vasquez C, Mores CN, Wearing HJ. Modeling mosquito-borne disease spread in U.S. urbanized areas: the case of dengue in Miami. PLoS ONE. 2016;11:e0161365.

13. Goindin D, Delannay C, Ramdini C, Gustave J, Fouque F. Parity and longevity of Aedes aegypti according to temperatures in controlled conditions and consequences on dengue transmission risks. PLOS ONE. 2015;10:e0135489.

14. Hardy JL, Houk EJ, Kramer LD, Reeves WC. Intrinsic factors affecting vector competence of mosquitoes for arboviruses. Annu Rev Entomol. 1983;28:229-62.

15. Souza-Neto JA, Powell JR, Bonizzoni M. Aedes aegypti vector competence studies: a review. Infect Genet Evol. 2019;67:191-209.

16. Lounibos LP, Kramer LD. Invasiveness of Aedes aegypti and Aedes albopictus and vectorial capacity for chikungunya virus. J Infect Dis. 2016;214(Suppl. 5):S453-8.

17. Macdonald G. The epidemiology and control of malaria. London: Oxford University Press; 1957.

18. Garrett-Jones C. Prognosis for interruption of malaria transmission through assessment of the mosquito's cectorial capacity. Nature. 1964:204:1173-5

19. Bagny L, Delatte H, Quilici S, Fontenille D. Progressive decrease in Aedes aegypti distribution in Reunion Island since the 1900s. J Med Entomol. 2009;46:1541-5.

20. Tsetsarkin KA, Vanlandingham DL, McGee CE, Higgs S. A single mutation in chikungunya virus affects vector specificity and epidemic potential. PLoS Pathog. 2007;3:e201.

21. Vazeille M, Moutailler S, Coudrier D, Rousseaux C, Khun H, Huerre M, et al. Two chikungunya isolates from the outbreak of La Reunion (Indian Ocean) exhibit different patterns of infection in the mosquito, Aedes albopictus. PLOS ONE. 2007;2:e1168.

22. Kilpatrick AM, Meola MA, Moudy RM, Kramer LD. Temperature, viral genetics, and the transmission of West Nile virus by Culex pipiens mosquitoes. PLoS Pathog. 2008;4:e1000092.

23. Ciota AT, Kramer LD. Vector-virus interactions and transmission dynamics of West Nile virus. Viruses. 2013;5:3021-47.

24. Moudy RM, Meola MA, Morin LL, Ebel GD, Kramer LD. A newly emergent genotype of West Nile virus is transmitted earlier and more efficiently by Culex mosquitoes. Am J Trop Med Hyg. 2007;77:365-70. 
25. Dye $C$. The analysis of parasite transmission by bloodsucking insects. Annu Rev Entomol. 1992;37:1-19.

26. Ruckert C, Ebel GD. How do virus-mosquito interactions lead to viral emergence? Trends Parasitol. 2018;34(4):310-21.

27. Petersen MT, Silveira IDD, Tatila-Ferreira A, David MR, Chouin-Carneiro T, Van den Wouwer $L$, et al. The impact of the age of first blood meal and Zika virus infection on Aedes aegypti egg production and longevity. PLoS One. 2018;13:e0200766.

28. Christofferson RC, Mores CN. Estimating the magnitude and direction of altered arbovirus transmission due to viral phenotype. PLoS ONE. 2011;6:e16298.

29. Smith DL, McKenzie FE. Statics and dynamics of malaria infection in Anopheles mosquitoes. Malar J. 2004;3:13.

30. Massad E, Coutinho FA. Vectorial capacity, basic reproduction number, force of infection and all that: formal notation to complete and adjust their classical concepts and equations. Mem Inst Oswaldo Cruz. 2012;107:564-7.

31. Dye C. Vectorial capacity: must we measure all its components? Parasitol Today. 1986;2:203-9.

32. Smith DL, Battle KE, Hay SI, Barker CM, Scott TW, McKenzie FE. Ross, macdonald, and a theory for the dynamics and control of mosquitotransmitted pathogens. PLoS Pathog. 2012;8:e1002588.

33. Garrett-Jones C, Shidrawi GR. Malaria vectorial capacity of a population of Anopheles gambiae: an exercise in epidemiological entomology. Bull World Health Organ. 1969;40:531-45.

34. Styer LM, Carey JR, Wang JL, Scott TW. Mosquitoes do senesce: departure from the paradigm of constant mortality. Am J Trop Med Hyg. 2007;76:111-7.

35. Novoseltsev VN, Michalski Al, Novoseltseva JA, Yashin Al, Carey JR, Ellis AM. An age-structured extension to the vectorial capacity model. PLoS ONE. 2012;7:e39479.

36. Rock KS, Wood DA, Keeling MJ. Age- and bite-structured models for vector-borne diseases. Epidemics. 2015;12:20-9.

37. Kawiecki AB, Mayton EH, Dutuze MF, Goupil BA, Langohr IM, Del Piero F, et al. Tissue tropisms, infection kinetics, histologic lesions, and antibody response of the MR766 strain of Zika virus in a murine model. Virol J. 2017;14:82

38. Ciota AT, Bialosuknia SM, Zink SD, Brecher M, Ehrbar DJ, Morrissette MN, et al. Effects of Zika virus strain and Aedes mosquito species on vector competence. Emerg Infect Dis. 2017;23:1110-7.

39. Richards SL, Pesko K, Alto BW, Mores CN. Reduced infection in mosquitoes exposed to blood meals containing previously frozen flaviviruses. Virus Res. 2007;129:224-7.

40. Tramonte AR, Christofferson RC. Investigating the probability of establishment of Zika virus and detection through mosquito surveillance under different temperature conditions. PLOS ONE. 2019;14:e0214306.

41. Tesla B, Demakovsky LR, Mordecai EA, Ryan SJ, Bonds MH, Ngonghala CN, et al. Temperature drives Zika virus transmission: evidence from empirical and mathematical models. Proc Biol Sci. 2018;285:20180795.

42. R Development Core Team. R: a language and environment for statistical computing. Vienna: R Foundation for Statistical Computing; 2018. https:// www.R-project.org/.

43. Paaijmans KP, Cator $L$, Thomas MB. Temperature-dependent prebloodmeal period and temperature-driven asynchrony between parasite development and mosquito biting rate reduce malaria transmission intensity. PLoS ONE. 2013;8:e55777.

44. Christofferson RC, Mores CN, Wearing HJ. Characterizing the likelihood of dengue emergence and detection in naive populations. Parasit Vectors. 2014;7:282.

45. Zermoglio PF, Robuchon E, Leonardi MS, Chandre F, Lazzari CR. What does heat tell a mosquito? Characterization of the orientation behaviour of Aedes aegypti towards heat sources. J Insect Physiol. 2017;100:9-14.

46. Styer LM, Kent KA, Albright RG, Bennett CJ, Kramer LD, Bernard KA. Mosquitoes inoculate high doses of West Nile virus as they probe and feed on live hosts. PLoS Pathog. 2007;3:1262-70.

47. Matsuoka H, Yoshida S, Hirai M, Ishii A. A rodent malaria, Plasmodium berghei, is experimentally transmitted to mice by merely probing of infective mosquito, Anopheles stephensi. Parasitol Int. 2002:51:17-23.
48. Dubrulle M, Mousson L, Moutailler S, Vazeille M, Failloux AB. Chikungunya virus and Aedes mosquitoes: saliva is infectious as soon as two days after oral infection. PLoS ONE. 2009:4:e5895.

49. Paaijmans KP, Blanford S, Chan BH, Thomas MB. Warmer temperatures reduce the vectorial capacity of malaria mosquitoes. Biol Lett. 2012;8:465-8

50. Kucharski AJ, Funk S, Eggo RM, Mallet HP, Edmunds WJ, Nilles EJ. Transmission dynamics of Zika virus in island populations: a modelling analysis of the 2013-14 French Polynesia outbreak. PLoS Negl Trop Dis. 2016;10:e0004726.

51. Styer LM, Minnick SL, Sun AK, Scott TW. Mortality and reproductive dynamics of Aedes aegypti (Diptera: Culicidae) fed human blood. Vector Borne Zoonotic Dis. 2007;7:86-98.

52. Perkasa A, Yudhaputri F, Haryanto S, Hayati RF, Ma'roef CN, Antonjaya U, et al. Isolation of Zika virus from febrile patient, Indonesia. Emerg Infect Dis. 2016;22:924-5.

53. Waggoner JJ, Gresh L, Vargas MJ, Ballesteros G, Tellez Y, Soda KJ, et al. Viremia and clinical presentation in Nicaraguan patients infected with Zika virus, chikungunya virus, and dengue virus. Clin Infect Dis. 2016;63:1584-90

54. Carrington LB, Armijos MV, Lambrechts L, Scott TW. Fluctuations at a low mean temperature accelerate dengue virus transmission by Aedes aegypti. PLoS Negl Trop Dis. 2013;7:e2190.

55. Alto BW, Wiggins K, Eastmond B, Ortiz S, Zirbel K, Lounibos LP. Diurnal temperature range and chikungunya virus infection in invasive mosquito vectors. J Med Entomol. 2018;55:217-24.

56. Muttis E, Balsalobre A, Chuchuy A, Mangudo C, Ciota AT, Kramer LD, et al. Factors related to Aedes aegypti (Diptera: Culicidae) populations and temperature determine differences on life-history traits with regional implications in disease transmission. J Med Entomol. 2018;55:1105-12.

57. Armstrong PM, Ehrlich HY, Magalhaes T, Miller MR, Conway PJ, Bransfield $A$, et al. Successive blood meals enhance virus dissemination within mosquitoes and increase transmission potential. Nat Microbiol. 2020;5:239-47

58. Sikulu-Lord MT, Milali MP, Henry M, Wirtz RA, Hugo LE, Dowell FE, et al. Near-infrared spectroscopy, a rapid method for predicting the age of male and female wild-type and Wolbachia infected Aedes aegypti. PLoS Negl Trop Dis. 2016;10:e0005040.

59. Lambert B, Sikulu-Lord MT, Mayagaya VS, Devine G, Dowell F, Churcher TS. Monitoring the age of mosquito populations using near-infrared spectroscopy. Sci Rep. 2018;8:5274.

60. Gonzalez Jimenez M, Babayan SA, Khazaeli P, Doyle M, Walton F, Reedy E, et al. Prediction of mosquito species and population age structure using mid-infrared spectroscopy and supervised machine learning. Wellcome Open Res. 2019;4:76.

61. Johnson BJ, Hugo LE, Churcher TS, Ong OTW, Devine GJ. Mosquito age grading and vector-control programmes. Trends Parasitol. 2020;36:39-51.

62. Onyango MG, Bialosuknia SM, Payne AF, Mathias N, Kuo L, Vigneron A et al. Increased temperatures reduce the vectorial capacity of Aedes mosquitoes for Zika virus. Emerg Microbes Infect. 2020;9:67-77.

63. Althouse BM, Hanley KA. The tortoise or the hare? Impacts of within-host dynamics on transmission success of arthropod-borne viruses. Philos Trans R Soc Lond B Biol Sci. 2015. https://doi.org/10.1098/rstb.2014.0299.

64. Pena-Garcia VH, Christofferson RC. Correlation of the basic reproduction number (R0) and eco-environmental variables in Colombian municipalities with chikungunya outbreaks during 2014-2016. PLoS Negl Trop Dis. 2019;13:e0007878.

65. Goddard LB, Roth AE, Reisen WK, Scott TW. Vector competence of California mosquitoes for West Nile virus. Emerg Infect Dis. 2002;8:1385-91.

\section{Publisher's Note}

Springer Nature remains neutral with regard to jurisdictional claims in published maps and institutional affiliations. 